\title{
Quality management system for screening esophagogastroduo- denoscopy improves detection of Helicobacter pylori-negative interval gastric cancer
}

\section{(ㄷ)(우)}

\author{
Authors \\ Institutions \\ 1 Koganei Tsurukame Clinic, Endoscopy Center, Tokyo, \\ Japan \\ 2 Shinjuku Tsurukame Clinic, Digestive Disease Center, \\ Tokyo, Japan \\ 3 Mirraza Shinjuku Tsurukame Clinic, Tokyo, Japan \\ 4 Yotsuya Medical Cube, Endoscopy Center, Tokyo, Japan
}

Fumiaki Ishibashi ${ }^{1,2}$, Konomi Kobayashi ${ }^{1}$, Tomohiro Kawakami ${ }^{1}$, Ryu Tanaka ${ }^{2}$, Kazuaki Sugihara ${ }^{2,3}$, Satoshi Baba ${ }^{2,4}$

submitted 5.5.2021

accepted after revision 13.8 .2021

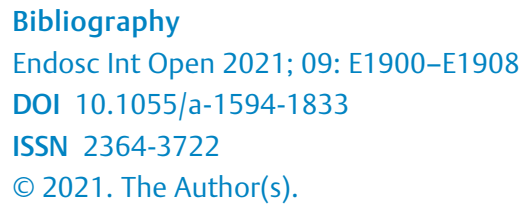
Commons Attribution-NonDerivative-NonCommercial License, permitting copying and reproduction so long as the original work is given appropriate credit. Contents may not be used for commercial purposes, or adapted, remixed, transformed or built upon. (https://creativecommons.org/licenses/by-nc-nd/4.0/)

Georg Thieme Verlag KG, Rüdigerstraße 14,

70469 Stuttgart, Germany

\section{Corresponding author}

Fumiaki Ishibashi, MD, PhD, Koganei Tsurukame Clinic, Endoscopy Center, 6-14-28-3F, Honcho, Koganei-shi, Tokyo, Japan, 184-0004

Fax: +81-42-386-7390

ishibashi@tsurukamekai.jp

\section{ABSTRACT}

Background and study aims Double-checking the findings of examinations is necessary for endoscopy quality control in gastric cancer screening; however, there have been no reports showing its effectiveness. We prospectively analyzed the effectiveness of a quality management system (QMS) in endoscopy for gastric cancer screening.

Patients and methods QMS was defined as having images and reports checked by a second endoscopist on the same day and reporting inconsistencies to the examining endoscopist. Patients diagnosed with early gastric cancer (EGC) in the 2 years before and after the introduction of QMS were divided into two groups: the interval cancer group, which included those for whom cancer was detected within 1 year of the last endoscopy and the noninterval cancer group. Changes in detection rates were compared.

Results Before the introduction of QMS, 11 interval EGC cases were diagnosed among 36,189 endoscopies, whereas after the introduction, 32 interval ECG cases were diagnosed among 38,290 endoscopies $(P=0.004)$. Fifteen noninterval EGC cases were diagnosed before the introduction, while 12 noninterval EGC cases were diagnosed after the introduction; no significant difference was observed. Subanalyses by Helicobacter pylori (HP) infection status revealed no difference in the detection rate among HP-positive EGC patients, but the detection rates among HP-eradicated and HP-naïve EGC patients were improved $(P=0.005$ and $P=$ $0.011)$. Logistic regression analysis showed that QMS was an independent predictor for detection of HP-negative interval EGC ( $P=0.017, \mathrm{OR}=4.4,95 \% \mathrm{Cl}: 2.0-9.7)$.

Conclusions QMS improved the detection rate for HPnegative interval early gastric cancer. (UMIN000042991)

\section{Introduction}

Recent studies have revealed the superiority of esophagogastroduodenoscopy (EGD) in detecting gastric lesions at earlier stages compared with the barium swallowing test [1, 2], and EGD has been performed for early gastric cancer (EGC) screening worldwide. Since EGD has become the preferred method for screening for EGCs in healthy asymptomatic adults at health check-ups, especially in Eastern countries such as Japan and Korea [3-5], ensuring its quality has become increasingly important. Consequently, the guidelines published by relevant societies note the importance of quality control in EGD [6-8]. While several quality indicators for colonoscopy, including observation time, bowel preparation degree, and polyp detection 
rate, have been reported [9-13] and training methods aimed at improving these quality indicators have been established [14, $15]$, only a few studies on quality indicators in detection of EGCs, such as gastric observation time and biopsy rate, have been reported [16-20]. We have reported that the observation time of the stomach might be a quality indicator for detection of Helicobacter pylori (HP)-negative high-risk lesions, including EGC [20]; however, it is difficult to precisely manage the observation time in daily EGD-based screening because the time it takes to clean mucus and residue varies among patients, and the methods of observing the stomach are not uniform among endoscopists and institutions.

The interval between EGD and newly identified gastric cancer has been discussed, and the concept of interval gastric cancer (IGC) has been proposed [21-23]. While the definition of IGC is not precise, several reports define it as gastric cancer found within 1 to 5 years of the most recent EGD [21-24]. These reports revealed that IGC lesions were relatively small and undifferentiated (diffuse-type gastric cancers) [23, 24]. Because gastric cancer is common in Eastern countries such as Japan and Korea due to the high rate of HP infection, these countries have established nationwide EGC screening programs (population-based screening). Early detection of EGC as IGC is important for gastric cancer screening programs, and the detection rate for interval cancer is expected to be a quality indicator. However, the methodology required to achieve this goal has not been established.

We hypothesized that standardizing the nonquantitative skills of endoscopists, such as attention to small lesions and overall concentration during examinations, was effective in identifying IGCs with a short interval. Here, we introduced a quality management system (QMS) for screening EGD to monitor and manage the nonquantitative skills of endoscopists and prospectively analyzed the efficacy of this system in detection of IGCs during EGD-based screening. This study aimed to reveal whether introducing QMS can improve identification of unclear or small lesions, which are common characteristics of HP-eradicated and HP-naïve EGC, by ensuring the quality of the initial examination.

\section{Patients and methods}

\section{Study design}

This prospective, observational cohort study was approved by the Ethics Committee of Shinjuku Tsurukame Clinic (Approval number: 1902) on August 14, 2018, and registered in the University Hospital Medical Information Network Clinical Trials Registry (UMIN-CTR) (Registration number: UMIN000042991). The intervention implemented in this study was introduction of QMS. The cohorts included in this study were the QMS cohort (intervention cohort) with feedback to the examining physician by the QMS and the control cohort without feedback by the QMS. The endoscopists and patients in the control cohort were completely independent of the QMS cohort, a population that did not receive any interventions in this study.

\section{Subjects}

Patients who underwent upper gastrointestinal endoscopy at Shinjuku Tsurukame Clinic (located in the central urban area of Tokyo) or Koganei Tsurukame Clinic (located in a suburban city further to the west, within Tokyo Metropolis) as part of a health checkup or outpatient care and who underwent subsequent examination in the same facility within the past 5 years were enrolled in this study (from August 2016 to September 2020). Patients with a history of surgical gastrectomy were excluded. Those who were unable to complete the endoscopy for any reason were excluded.

\section{Quality management system for screening esophagogastroduodenoscopy}

The QMS was introduced in August 2018, and the change in the gastric cancer detection rate in the 2 years before and after introduction of the system was analyzed. In this study, the first 2 years prior to implementation of the QMS are referred to as the first period and the 2 years after the implementation of the QMS are referred to as the second period. The QMS is performed in two steps. First, the endoscopist writes a report on the findings from the examination as soon as it is completed and temporarily stores the report with all the relevant images. A second endoscopist then reviews the stored report and images for each patient to ensure that the report is accurate and that the entire stomach was comprehensively observed. The comprehensiveness of the images was assessed according to whether each part of the stomach (anterior and posterior wall; lesser and greater curvature of the cardia; upper, middle and lower portions; and angle and antrum; at least 24 images) was appropriately captured. If there were any mistakes in the written report or the images were insufficient, the report was sent back to the initial endoscopist. The endoscopists who performed the double-check were certified by the Japan Gastrointestinal Endoscopy Society (JGES). The QMS was introduced to all endoscopists in the QMS cohort in August 2018. The population eligible for QMS was defined as the QMS cohort. Endoscopies performed by endoscopists who performed doublechecking in the QMS were not included in the QMS cohort, but were treated independently as the control cohort. The endoscopists in each cohort were completely independent of each other, and there was no difference in the background of the endoscopists.

\section{Endoscopic procedures}

All physicians had been trained based on the program of the JGES and were board-certified member of the organization at the start of this study (August 2016). The method and technique for stomach observation were standardized; mucus from the whole stomach was carefully removed with cleaning solution including mucolytic agents (dimethicone), and each part of the stomach from the cardia to the antrum was observed. The duration of observation of the stomach was calculated after examination of the stored endoscopic images. The endoscopists were required to perform biopsy after observing the whole stomach, and the time spent performing biopsy was not 
included in the observation time. Sedative agents (pethidine hydrochloride, midazolam and propofol) were administered at patient request. The endoscopy devices used were the GIFQ260, GIF-H290 and GIF-H290Z (Olympus, Tokyo, Japan). The processor used was the EVIS LUCERA ELITE (Olympus, Tokyo, Japan). No changes were made to the endoscopy equipment within the study period.

\section{HP infection status}

All gastric cancer patients were investigated in detail for HP infection status. For cases in which gastric cancer was not detected, HP infection status was investigated for cases with endoscopically active gastritis or atrophic gastritis, but for cases suspected to be HP-negative based on endoscopic findings, the patients were judged to be HP-negative without additional testing. HP infection was defined based on a positive result on the serum anti-HP-IgG antibody, urea breath or stool HP antigen test, and endoscopic findings of diffuse redness of the stomach, increased mucus volume, and thickening of the folds on the greater curvature, which are characteristics of current infection. HP positivity was diagnosed with HP antibody $\geq 10 \mathrm{U} /$ $\mathrm{mL}$ or with urea breath test $\geq 2.5 \%$. Successful eradiation of HP was defined as a urea breath test result $<2.5 \%$ performed more than 2 months after completion of eradication medication. HPnaïvety was defined as meeting at least two of the following criteria: anti HP-IgG antibody $<3 \mathrm{U} / \mathrm{mL}$, urea breath test $<2.5 \%$, negative HP antigen in stool, and negative HP bacillus on direct specular examination of biopsy specimen by Giemsa staining. Patients with successful eradication of HP and HP-naïve patients were regarded as HP-negative patients in this study.

\section{Data analyses}

The primary endpoint of this study was the detection rate for interval cancers before and after introduction of QMS. The interval cancers were defined as gastric cancers newly identified within 1,3 , or 5 years of the last endoscopy, according to previous reports [21-24]. For the analysis of interval cancer, the detection rate was calculated using patients with endoscopic experience within 5 years as the denominator. For the analysis of all EGCs and advanced gastric cancers (AGCs) detected during the study period, the detection rate was calculated for all patients, including those who had no endoscopic experience at the same institution within 5 years. All statistical analyses were performed with R 3.3.3 [25]. The average values were compared between the two groups with a Student's $t$-test. Categorical variables were compared with the chi-square test. Logistic regression analysis was performed to predict whether HPnegative gastric cancer would manifest as an interval cancer, and odds ratios and $95 \%$ confidential intervals were calculated for significant variables. $P<0.05$ was regarded as statistically significant.

\section{Results}

\section{Impact of the quality management system on the detection of gastric cancer}

A total of 49,752 and 51,861 patients in the QMS cohort and a total of 9,112 and 9,513 patients in the control cohort underwent endoscopy before and after the implementation of the quality management protocol, respectively. Of those, 36,189 and 38,290 patients in the QMS cohort and 6,910 and 7,214 patients in the control cohort underwent at least one more endoscopies within 5 years and were regarded as eligible for this study ( $\triangleright$ Fig. 1). In the QMS cohort, 26 endoscopists performed examinations in the first and second period, and in the control cohort, five endoscopists performed examinations in the first and second period. Twenty of the 26 endoscopists were the same in the first and second periods. There was no difference in background factors for any patients in the first and second periods ( Table $\mathbf{1}$ ). While detection rates for EGC and AGC were not significantly different between the two periods $(P=$ 0.063 and $P=0.963$, respectively), the detection rate for interval EGC significantly increased after introduction of the QMS

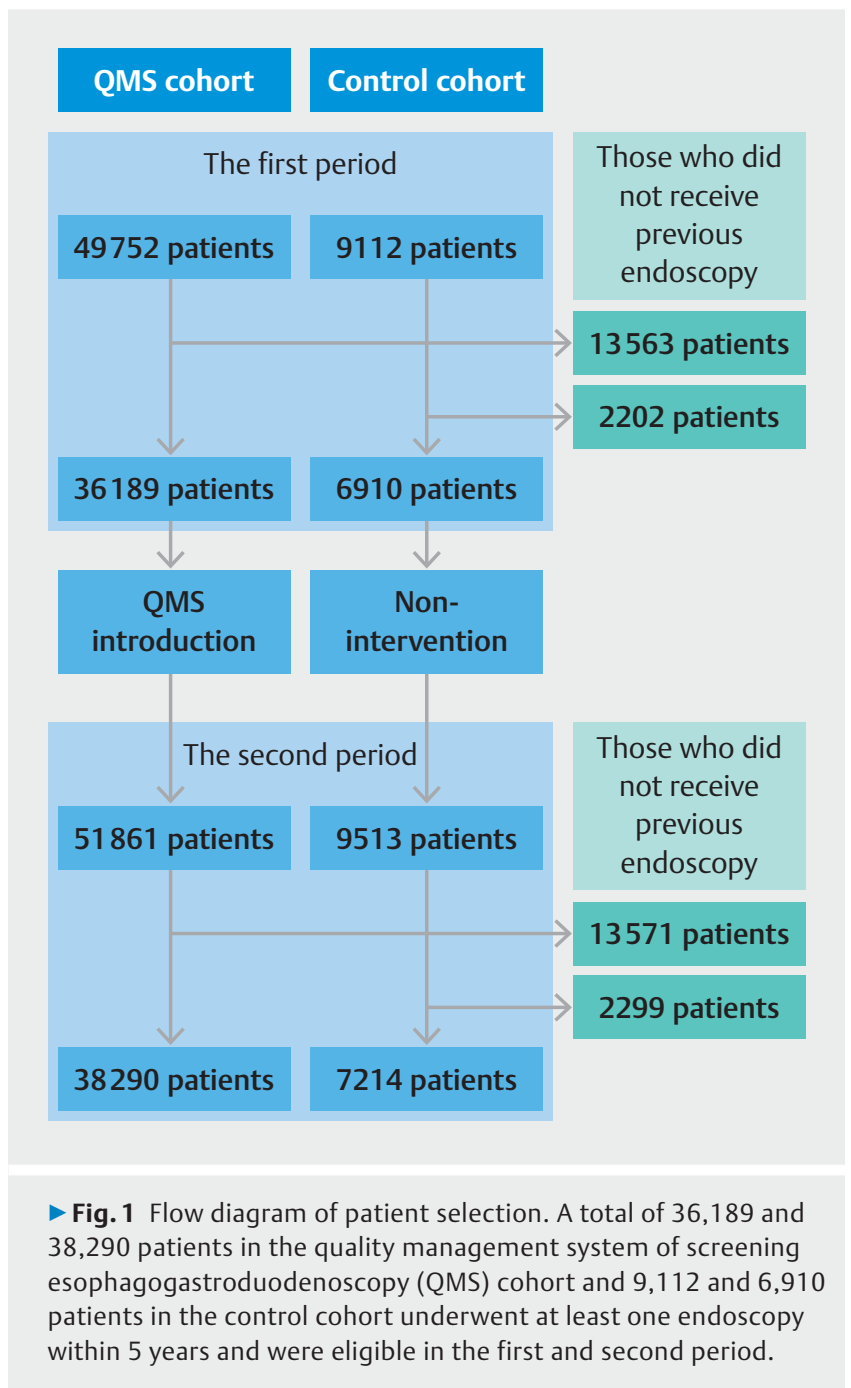


- Table 1 Background characteristics of patients enrolled in the study.

\begin{tabular}{|c|c|c|c|}
\hline All patients & $\begin{array}{l}\text { First period } \\
(n=58,864)\end{array}$ & $\begin{array}{l}\text { Second period } \\
(n=61,374)\end{array}$ & $P$ value \\
\hline Age (years) & $50.4 \pm 4.6$ & $50.8 \pm 4.1$ & 0.774 \\
\hline Sex (male:female) & $1.7: 1$ & $1.7: 1$ & 1.000 \\
\hline BMI & $22.1 \pm 1.7$ & $22.4 \pm 2.2$ & 0.875 \\
\hline Asymptomatic:symptomatic & $22: 1$ & $21: 1$ & 0.773 \\
\hline Use of sedative agents (\%) & $34,561(58.7 \%)$ & $36,290(59.1 \%)$ & 0.144 \\
\hline Smoking habit (\%) & $5,419(9.2 \%)$ & $5,517(9.0 \%)$ & 0.195 \\
\hline Patients eligible for analysis of interval cancer & $\begin{array}{l}\text { First period } \\
(n=43,099)\end{array}$ & $\begin{array}{l}\text { Second period } \\
(n=45,504)\end{array}$ & Pvalue \\
\hline Age (years) & $50.6 \pm 4.2$ & $50.4 \pm 3.7$ & 0.825 \\
\hline Sex (male:female) & $1.7: 1$ & $1.7: 1$ & 1.000 \\
\hline BMI & $22.3 \pm 1.8$ & $22.2 \pm 2.6$ & 0.896 \\
\hline Asymptomatic:symptomatic & $22: 1$ & $21: 1$ & 0.688 \\
\hline Use of sedative agents (\%) & $26,032(60.4 \%)$ & $27,666(60.8 \%)$ & 0.228 \\
\hline Smoking habit (\%) & $3,922(9.1 \%)$ & $4,050(8.9 \%)$ & 0.305 \\
\hline
\end{tabular}

( $>$ Table 2). Of note, detection rates for interval EGC diagnosed within 1,3 , and 5 years all increased significantly $(0.030 \%$ vs $0.084 \%, 0.030 \%$ vs $0.091 \%$ and $0.041 \%$ vs $0.094 \%$ in the first and second periods, $P=0.004, P=0.008$ and $P=0.009$, respectively) ( $\triangleright$ Table 2 ). On the other hand, in the control cohort, the detection rate for interval EGC did not change before and after introduction of the QMS $(0.029 \%$ vs $0.021 \%, 0.029 \%$ vs $0.042 \%$ and $0.043 \%$ vs $0.055 \%, P=1.000, P=1.000$ and $P=$ 1.000 , respectively). Of the $31 \mathrm{HP}$-positive EGCs, 25 were diagnosed with $\mathrm{HP}$ antibody $\geq 10 \mathrm{U} / \mathrm{mL}$ and six with urea breath test $\geq 2.5 \%$. All cases of HP-naïve EGC were confirmed as HPnegative by having anti-HP-IgG antibody $<3 \mathrm{U} / \mathrm{mL}$. In addition, negative UBT in 15 of 17 cases and negative HP antigen in stool in two cases met the HP-negative criteria in the study. All HPnaïve EGC cases had endoscopically visible regular arrangement collecting venules and no mucosal atrophy. EGC characteristics during the whole study period were not different in the first and second period ( $\triangleright$ Table 3 ).

\section{Effect of the quality management system on quality of inspection}

To address whether the QMS had a positive impact on the quality of each endoscopy and all endoscopies, we compared the number of times that the second endoscopist reported different examination or image findings during the quality management procedure. In total, $28.8 \%$ of the feedback received during the study period was related to the quality of the images (insufficient cleaning, halos, insufficient air volume), $14.4 \%$ was related to the comprehensiveness of the images, and $56.8 \%$ was related to the description in the report; no new lesions were found in any patient during the quality management process. The average amount of feedback decreased over time after introduction of the QMS and improved to $<1 \%$ after 8 weeks ( Fig.2). However, objective indicators, including the number of images per examination and the time required to observe the stomach, did not differ after introduction of QMS $(P=0.066$ and $P=0.119)$ ( $>$ Table 4). Fourteen cases with endoscopic images that were difficult to distinguish between gastritis and EGC were reexamined 1 year later, but there were no new gastric cancers found as a result.

\section{Analysis of identified interval early gastric cancers}

We further analyzed changes in detection of interval EGCs by HP infection status in the first and second periods. There was no change in the number of whole EGCs found in the first and second periods, when classified by HP infection status ( $\downarrow$ Table 5). However, while the detection rate for HP-positive interval EGCs was not different after introduction of QMS, the detection rate for HP-eradicated and -naïve interval EGCs significantly improved after introduction of QMS ( $>$ Table 5). Therefore, we included patients with HP-eradicated and HP-naïve EGC in the HP-negative EGC group and further analyzed the effect of QMS on detection of HP-negative interval EGCs by logistic regression. The analysis revealed that the number of endoscopies performed within 5 years $(P=0.021, \mathrm{OR}=3.7,95 \% \mathrm{Cl}: 1.2-11.5)$ and the introduction of QMS $(P=0.017, \mathrm{OR}=4.36,95 \% \mathrm{Cl}$ : 2.0-9.7) were independent predictors for detection of HP-negative interval EGCs ( $\triangleright$ Table 6$)$. 
- Table 2 Gastric cancer detection rates before and after introduction of the quality management system.

\begin{tabular}{|c|c|c|c|}
\hline QMS cohort & First period & Second period & $P$ value \\
\hline Analysis of interval cancer & $\mathrm{n}=36,189$ & $\mathrm{n}=38,290$ & \\
\hline - Interval cancer (within 1 year) (\%) & $11(0.030 \%)$ & $32(0.084 \%)$ & 0.004 \\
\hline - Interval cancer (within 3 year) (\%) & $14(0.030 \%)$ & $35(0.091 \%)$ & 0.008 \\
\hline - Interval cancer (within 5 year) (\%) & $15(0.041 \%)$ & $36(0.094 \%)$ & 0.009 \\
\hline Analysis of all identified cancer & $\mathrm{n}=49,752$ & $\mathrm{n}=51,861$ & \\
\hline - EGC (\%) & $26(0.052 \%)$ & $44(0.085 \%)$ & 0.063 \\
\hline - Sedative agent use (\%) & $18(69.2 \%)$ & $32(72.7 \%)$ & 0.789 \\
\hline $\begin{array}{l}\text { - HP infection status } \\
\text { (positive:eradicated:naïve) }\end{array}$ & $14: 8: 4$ & $12: 19: 13$ & 0.078 \\
\hline - Size $(\mathrm{mm})$ & $14.2 \pm 5.2$ & $12.9 \pm 4.6$ & 0.451 \\
\hline - Location (upper, middle, lower) & $6: 11: 9$ & $11: 20: 13$ & 0.907 \\
\hline - Type (protruded, depressed) & $9: 17$ & 19:25 & 0.615 \\
\hline - Pathology (differentiated, undifferentiated, GA-FG or fundic type) & 18:6:2 & $27: 8: 9$ & 0.359 \\
\hline - $\operatorname{AGC}(\%)$ & $3(0.006 \%)$ & $2(0.004 \%)$ & 0.963 \\
\hline Control cohort & First period & Second period & $P$ value \\
\hline Analysis of interval cancer & $n=6,910$ & $n=7,214$ & \\
\hline - Interval cancer (within 1 year) (\%) & $2(0.029 \%)$ & $2(0.021 \%)$ & 1.000 \\
\hline - Interval cancer (within 3 years) (\%) & $2(0.029 \%)$ & $3(0.042 \%)$ & 1.000 \\
\hline - Interval cancer (within 5 years) (\%) & $3(0.043 \%)$ & $4(0.055 \%)$ & 1.000 \\
\hline Analysis of all identified cancer & $n=9,112$ & $n=9,513$ & \\
\hline - EGC (\%) & $4(0.058 \%)$ & $5(0.069 \%)$ & 1.000 \\
\hline - Sedative agent use (\%) & $3(75.0 \%)$ & $3(60.0 \%)$ & 1.000 \\
\hline $\begin{array}{l}\text { - HP infection status } \\
\quad \text { (positive, eradicated, naïve) }\end{array}$ & $2: 2: 0$ & $3: 2: 0$ & NA \\
\hline - Size $(\mathrm{mm})$ & $13.2 \pm 3.8$ & $14.1 \pm 3.6$ & 0.866 \\
\hline - Location (upper, middle, lower) & $0: 2: 2$ & $1: 3: 1$ & 0.487 \\
\hline - Type (protruded, depressed) & $1: 3$ & $1: 4$ & 1.000 \\
\hline - Pathology (differentiated, undifferentiated, GA-FG or fundic type) & $3: 1: 0$ & $4: 1: 0$ & NA \\
\hline - $\operatorname{AGC}(\%)$ & $1(0.014 \%)$ & $0(0 \%)$ & 0.489 \\
\hline
\end{tabular}

QMS, quality management system; EGC, early gastric cancer; HP, Helicobacter pylori; AGC, advanced gastric cancer; GA-FG, gastric adenocarcinoma of fundic gland type; NA, not applicable. Size was expressed as mean \pm SD.

\section{Discussion}

In this study, we prospectively compared gastric cancer cases occurring in the 2 years before introduction of the QMS with newly detected gastric cancer cases after introduction of the QMS. EGD is widely performed to screen for gastric cancer in countries such as Japan and South Korea, and a program for population-based gastric cancer screening is being established under the leadership of local governments. In populationbased gastric cancer screening, double-checking of findings has been recommended to ensure the accuracy of the examining physician, but there have been no reports on specific meth- odologies or its effectiveness. This study is the first report on a QMS involving double-checking of findings that has been defined in detail and demonstrated to be effective. The ultimate goal of gastric cancer screening is to reduce cancer deaths by detecting gastric cancer early. However, as indications for endoscopic treatment are expanding, it is increasingly important to improve detection of EGC. In this study, we showed that our newly defined QMS is useful for early detection of the disease.

We defined QMS as having a subset of reports and images reviewed by a second endoscopist on the same day. This system is expected to rapidly improve the quality of examinations 
- Table 3 Background characteristics of patients whose early gastric cancer was identified before and after introduction of the quality management system.

\begin{tabular}{|c|c|c|c|}
\hline & $\begin{array}{l}\text { First period } \\
(n=26)\end{array}$ & $\begin{array}{l}\text { Second period } \\
(n=44)\end{array}$ & $P$ value \\
\hline Age (years) & $60.9 \pm 2.2$ & $60.6 \pm 1.4$ & 0.894 \\
\hline Sex (male:female) & $18: 8$ & 29:15 & 0.982 \\
\hline BMI & $22.8 \pm 2.7$ & $21.9 \pm 2.3$ & 0.912 \\
\hline Symptomatic:asymptomatic & $1: 25$ & $3: 41$ & 1.000 \\
\hline Use of sedative agents (\%) & $18(69.2 \%)$ & $29(65.9 \%)$ & 0.982 \\
\hline Smoking habit (\%) & $4(15.4 \%)$ & $6(13.6 \%)$ & 1.000 \\
\hline Number of endoscopies within 5 years (times) & $2.4 \pm 0.3$ & $2.8 \pm 0.1$ & 0.093 \\
\hline
\end{tabular}

- Table 4 Quality of examinations before and after introduction of the quality management system.

\begin{tabular}{|l|l|l|l|}
\hline & First period & Second period & P value \\
\hline Number of images per examination & $48.2 \pm 0.3$ & $49.1 \pm 0.5$ & 0.066 \\
\hline Stomach screening time (sec) & $213.7 \pm 0.9$ & $215.6 \pm 0.9$ & 0.119 \\
\hline Data were expressed as mean \pm SD. & & \\
\hline
\end{tabular}

- Table 5 Interval gastric cancer detection rate by HP infection status before and after introduction of the quality management system.

\begin{tabular}{|c|c|c|c|}
\hline & First period & Second period & $P$ value \\
\hline \multicolumn{4}{|c|}{ Interval cancer (within 1 year) } \\
\hline HP-positive & $7(0.920 \%)$ & $4(0.390 \%)$ & 0.762 \\
\hline HP-eradicated & $3(0.032 \%)$ & $17(0.170 \%)$ & 0.005 \\
\hline HP-naïve & $1(0.003 \%)$ & $11(0.027 \%)$ & 0.011 \\
\hline \multicolumn{4}{|c|}{ Interval cancer (within 3 years) } \\
\hline HP-positive & $7(0.920 \%)$ & $4(0.390 \%)$ & 0.762 \\
\hline HP-eradicated & $4(0.043 \%)$ & $19(0.170 \%)$ & 0.013 \\
\hline HP-naïve & $3(0.010 \%)$ & $13(0.027 \%)$ & 0.032 \\
\hline \multicolumn{4}{|c|}{ Interval cancer (within 5 years) } \\
\hline HP-positive & $7(0.920 \%)$ & $5(0.650 \%)$ & 0.762 \\
\hline HP-eradicated & $5(0.079 \%)$ & $18(0.270 \%)$ & 0.019 \\
\hline HP-naïve & $3(0.010 \%)$ & $13(0.042 \%)$ & 0.032 \\
\hline \multicolumn{4}{|c|}{ All identified EGC } \\
\hline HP-positive & $14(0.920 \%)$ & $12(0.390 \%)$ & 0.696 \\
\hline HP-eradicated & $8(0.032 \%)$ & $19(0.170 \%)$ & 0.055 \\
\hline HP-naïve & $4(0.003 \%)$ & $13(0.027 \%)$ & 0.051 \\
\hline
\end{tabular}

through rapid feedback to endoscopists. In fact, the frequency of feedback was halved in the first week after QMS was introduced. In addition, the frequency of feedback has been less than $1 \%$ since 8 weeks after introduction of the system; therefore, it can be expected that the system will be effective in maintaining a certain level of quality in examinations ( $>$ Fig. 2 ). 
- Table 6 Comparisons of background factors in patients with HP-negative interval and non-interval gastric cancers identified within 1 year.

\begin{tabular}{|c|c|c|c|c|c|c|}
\hline & $\begin{array}{l}\text { Interval } \\
\text { cancer } \\
(\mathbf{n}=32)\end{array}$ & $\begin{array}{l}\text { Non-interval } \\
\text { cancer } \\
(n=13)\end{array}$ & $\begin{array}{l}P \text { value } \\
\text { (univariate) }\end{array}$ & $\begin{array}{l}P \text { value } \\
\text { (multivariate) }\end{array}$ & OR & $95 \% \mathrm{Cl}$ \\
\hline Age (years) & $58.7 \pm 1.5$ & $60.1 \pm 3.0$ & 0.665 & 0.728 & & \\
\hline Sex (male:female) & $22: 10$ & $9: 4$ & 1.000 & 0.829 & & \\
\hline Size $(\mathrm{mm})$ & $8.2 \pm 0.9$ & $9.6 \pm 1.0$ & 0.358 & 0.057 & & \\
\hline Number of endoscopies & $3.6 \pm 0.2$ & $2.2 \pm 0.4$ & 0.001 & 0.021 & 3.7 & $1.2-11.5$ \\
\hline Symptomatic: Asymptomatic & $1: 31$ & $0: 13$ & 1.000 & 0.992 & & \\
\hline Location (U:M:L) & $10: 11: 11$ & $2: 5: 6$ & 0.534 & 0.356 & & \\
\hline Type (protruded:depressed) & $9: 23$ & $6: 7$ & 0.416 & 0.294 & & \\
\hline $\begin{array}{l}\text { Pathology } \\
\text { (Differentiated:undifferentiated:others) }\end{array}$ & $14: 9: 9$ & $9: 2: 2$ & 0.300 & 0.402 & & \\
\hline Physician's experience of endoscopy (year) & $11.9 \pm 0.5$ & $12.5 \pm 0.8$ & 0.490 & 0.684 & & \\
\hline Double check (+:-) & $27: 5$ & $5: 8$ & 0.007 & 0.017 & 4.4 & $2.0-9.7$ \\
\hline
\end{tabular}

HP, Helicobacter pylori; OR, odds ratio; $95 \% \mathrm{Cl}, 95 \%$ confidence interval. Data were expressed as mean \pm SD.

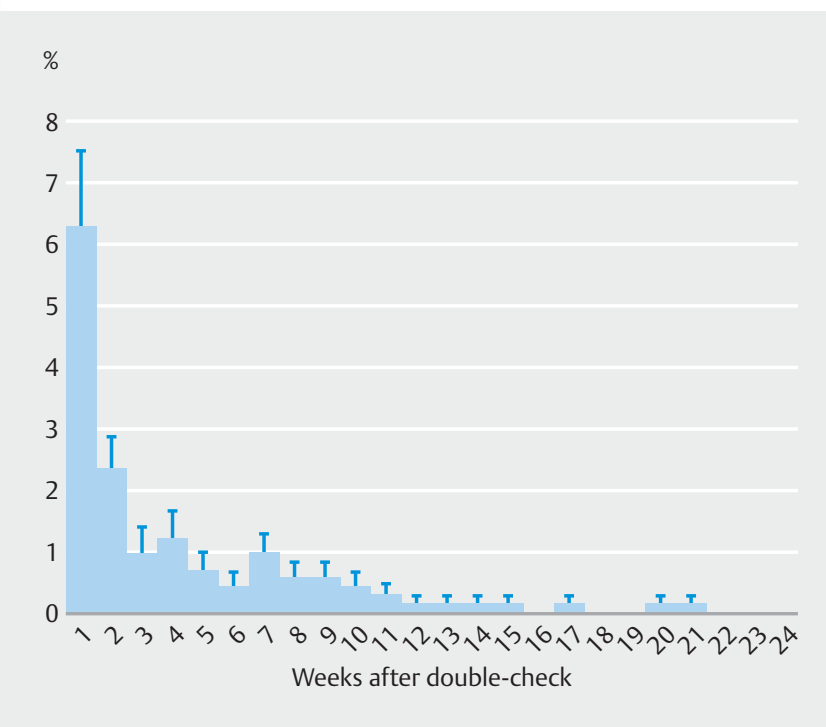

Fig. 2 Ratio of feedback provided to the initial endoscopists after introduction of the double-check system. Data are expressed as the mean \pm SEM of the feedback ratio.

Any new lesions were not found under QMS in this study. The reason for this is that all the endoscopists who were enrolled in this study were specialists who were board-certified members of JGES, and with regard to the photographs taken, there were no cases in which the lesions in the photographs were overlooked at the time of initial diagnosis. In fact, there was no change in detection in the first and second periods for lesions such as HP-positive gastric cancer, which is easily identified in QMS, and the effect of QMS was demonstrated in cases such as HP-negative gastric cancer, which cannot be detected without careful attention. The significance of QMS lies in the educa- tion of endoscopists to ensure the quality of examination in initial endoscopy.

Although there was no improvement in detection of EGCs as a whole due to the introduction of QMS, regardless of HP infection status, when the analysis was limited to detection of interval cancer, there was a difference in the effect of QMS depending on HP infection status. The reason for the effect of QMS introduction in HP-negative gastric cancers, limited to interval cancer, may be that relatively large lesions that are detected more than 5 years after the last endoscopy are easily found regardless of QMS introduction. In fact, when HP-eradicated cases and HP-naïve cases were detected as non-interval cancers, they tended to be larger than those detected as interval cancers, although the difference was not significant, as shown in $>$ Table 6. Interval EGCs detected in the first and second periods were analyzed separately by HP infection status, and detection of HP-positive interval EGCs did not differ. The reason for this may be that HP-positive EGC lesions are relatively large in diameter and easily visible, while most HP negative gastric cancers are small gastric cancers $(<10 \mathrm{~mm})[26,27]$, so the detection rate may have been sufficiently good even in the absence of QMS. In fact, the size of HP-positive gastric cancers detected during this study was larger than that of HP-negative gastric cancers $(15.7 \pm 4.4 \mathrm{~mm}$ vs $10.2 \pm 3.8 \mathrm{~mm}, P=0.003)$. In addition, there was no difference in detection of AGC, which is also large, or in the first and second periods. Detection of HP-eradicated interval EGC and HP-naïve interval EGC significantly improved after the introduction. It has been reported that HP-negative EGCs are characterized by small-diameter and obscure lesions $[26,28-30]$. We believe that the QMS was effective in maintaining endoscopist attention and identifying HP-negative EGC lesions during the examination; these lesions are small in diameter and obscure, and identification requires visualizing slight 
tonal differences and uneven changes compared with the background mucosa.

In this study, quantifiable indices such as the number of endoscopic photographs and the observation time did not change in the first and second periods ( $>$ Fig. 2 and $\triangleright$ Table 3 ). This result suggests that all the endoscopists who participated in this study had received uniform endoscopic training following the JGES program and had reached an acceptable level for these quantifiable indicators before the introduction of the QMS. The results of the logistic regression analysis are reliable because the predictors for detection of interval EGCs were analyzed considering no difference in observation time, which is considered a quality indicator for EGC detection [16-18, 20]. For nonquantifiable indicators, such as attention to minute changes, endoscopists are educated by personal experience; QMS established in this study is potentially useful for controlling nonquantifiable indicators.

It has been reported that EGCs are frequently missed by previous endoscopy, but can be treated because of their slow progression [31]. In fact, three of the 13 HP-naïve interval EGCs could be identified by reviewing the previous endoscopy photographs during the study period; however, we were not able to detect such lesions with the QMS. It may be useful to keep the QMS in operation, especially for detecting HP-naïve interval EGC.

This study involved several limitations. First, the fact that two non-contemporaneous time intervals were examined might be associated with a time bias, although physician experience with endoscopy was analyzed in a multivariate analysis. It is possible that endoscopists voluntarily became more aware of HP-naïve EGCs after the introduction of the QMS. In that case, the possibility that the improvement in detection of HP-naïve EGC was strongly influenced by the time bias cannot be denied. Second, $>$ Table 6 is an analysis of cases in which HP-eradicated and -naïve EGC were integrated, and not the results of separate analyses because there was an insufficient number of cases. Third, in this study, QMS was not interrupted in the study protocol, and it is unclear whether the effect of QMS is only temporary or whether it is effective when continued. Fourth, detection of HP-naïve EGC might be overestimated because the patients in the denominator were judged to be HP-naïve without qualitative examination for HP infection. In the future, more robust evidence should be established through comparative studies with controls without quality management in a randomized controlled trial.

\section{Conclusions}

In this study, we established that a QMS improved the detection of HP-negative interval gastric cancer in EGD-based screening.

\section{Competing interests}

The authors declare that they have no conflict of interest.

\section{References}

[1] Hamashima C, Ogoshi K, Okamoto M et al. A community-based, casecontrol study evaluating mortality reduction from gastric cancer by endoscopic screening in Japan. PLoS One 2013; 8: e79088-e79096

[2] Hamashima C, Shabana M, Okada K et al. Mortality reduction from gastric cancer by endoscopic and radiographic screening. Cancer Sci 2015; 106: 1744-1749

[3] Hosokawa O, Miyanaga T, Kaizaki Y et al. Decreased death from gastriccancer by endoscopic screening: association with a populationbased can-cer registry. Scand J Gastroenterol 2008; 43: 1112-1115

[4] Jun JK, Choi KS, Lee HY et al. Effectiveness of the Korean national cancerscreening program in reducing gastric cancer mortality. Gastroenterology 2017; 152: 1319-1328

[5] Noh CH, Lee E, Lee $\mathrm{GH}$ et al. Association of intensive endoscopic screening burden with gastric cancer detection. JAMA Netw Open 2021; 4: e2032542

[6] Yao K, Uedo N, Kamada T et al. Guidelines for endoscopic diagnosis of early gastric cancer. Dig Endosc 2020; 32: 663-698

[7] Evans JA, Chandrasekhara V, Chathadi KV. ASGE Standards of Practice Committee. et al. The role of endoscopy in the management of premalignant and malignant conditions of the stomach. Gastrointest Endosc 2015; 82: 1-8

[8] Hamashima C. Cancer screening guidelines and policy making: 15 years of experience in cancer screening guideline development in Japan. Jpn J Clin Oncol 2018; 48: 278-286

[9] Kaminski MF, Regula J, Kraszewska E et al. Quality indicators for colonoscopy and the risk of interval cancer. N Engl J Med 2010; 362: 1795-1803

[10] Rex DK, Petrini JL, Baron TH et al. Quality indicators for colonoscopy. Gastrointest Endosc 2006; 63: S16-S28

[11] Barclay RL, Vicari J], Doughty AS et al. Colonoscopic withdrawal times and adenoma detection during screening colonoscopy. N Engl J Med 2006; 355: 2533-2541

[12] Butterly L, Robinson CM, Anderson JC et al. Serrated and adenomatous polyp detection increases with longer withdrawal time: results from the New Hampshire Colonoscopy Registry. Am J Gastroenterol 2014; 109: 417-426

[13] Shaukat A, Rector TS, Church TR et al. Longer withdrawal time is associated with a reduced incidence of interval cancer after screening colonoscopy. Gastroenterology 2015; 149: 952-957

[14] Lim S, Hammond S, Park J et al. Training interventions to improve adenoma detection rates during colonoscopy: a systematic review and meta-analysis. Surg Endosc 2020; 34: 3870-3882

[15] Ishibashi F, Fukushima K, Kobayashi K et al. Individual feedback and monitoring of endoscopist performance improves the adenoma detection rate in screening colonoscopy: a prospective case-control study. Surg Endosc 2020; 35: 2566-2575

[16] Teh JL, Tan JR, Lau LJ et al. Longer examination time improves detection of gastric cancer during diagnostic upper gastrointestinal endoscopy. Clin Gastroenterol Hepatol 2015; 13: 480-487

[17] Kawamura T, Wada $\mathrm{H}$, Sakiyama $\mathrm{N}$ et al. Examination time as a quality indicator of screening upper gastrointestinal endoscopy for asymptomatic examinees. Dig Endosc 2017; 29: 569-575

[18] Park JM, Huo SM, Lee HH et al. Longer observation time increases proportion of neoplasms detected by esophagogastroduodenoscopy. Gastroenterology 2017; 153: 460-469

[19] Januszewicz W, Wieszczy P, Bialek A et al. The endoscopists' biopsy rate as a quality indicator for outpatient gastroscopy: a multicenter cohort study with validation. Gastrointest Endosc 2019; 89: 11411149 
[20] Ishibashi F, Kobayashi K, Fukushima K et al. Quality indicators for the detection of Helicobacter pylori-negative early gastric cancer: a retrospective observational study. Clin Endosc 2020; 53: 698-704

[21] Khalil Q, Gopalswamy N, Agrawal S. Missed esophageal and gastric cancers after esophagogastroduodenoscopy in a midwestern military veteran population. South Med J 2014; 107: 225-228

[22] Pimenta-Melo AR, Monteiro-Soares M, Libanio D et al. Missing rate for gastric cancer during upper gastrointestinal endoscopy: a systematic review and meta-analysis. Eur J Gastroenterol Hepatol 2016; 28: 1041-1049

[23] Park MS, Yoon JY, Chung HS et al. Clinicopathologic characteristics of interval gastric cancer in Korea. Gut Liver 2015; 9: 167-173

[24] Choi KS, Jun JK, Park EC et al. Performance of different gastric cancer screening methods in Korea: a population-based study. PLoS One 2012; 7: e50041

[25] R Core Team. A language and environment for statistical computing. R Foundation for Statistical Computing, Vienna, Austria. https://www. R-project.org/
[26] Sato C, Hirasawa K, Tateishi Y et al. Clinicopathological features of early gastric cancers arising in Helicobacter pylori uninfected patients. World J Gastroenterol 2020; 26: 2618-2631

[27] Nishizawa T, Suzuki H, Arano T et al. Characteristics of gastric cancer detected within 1 year after successful eradication of Helicobacter pylori. J Clin Biochem Nutr 2016; 59: 226-230

[28] Ueyama H, Matsumoto K, Nagahara A et al. Gastric adenocarcinoma of the fundic gland type (chief cell predominant type). Endoscopy 2014; 46: 153-157

[29] Ishibashi F, Fukushima K, Ito T et al. Influence of Helicobacter pylori Infection on endoscopic findings of gastric adenocarcinoma of the fundic gland type. J Gastr Cancer 2019; 19: 225-233

[30] Saka A, Yagi K, Nimura S. Endoscopic and histological features of gastric cancers after successful Helicobacter pylori eradication therapy. Gastric Cancer 2016; 19: 524-530

[31] Shimodate Y, Mizuno M, Doi A et al. Gastric superficial neoplasia: high miss rate but slow progression. Endosc Int Open 2017; 5: E722-E726

\section{CORRECTION}

Quality management system for screening esophagogastroduodenoscopy improves detection of Helicobacter pylori-negative interval gastric cancer

Fumiaki Ishibashi, Konomi Kobayashi, Tomohiro Kawakami et al.

Endoscopy International Open 2021; 09: E1900-E1908. DOI: 10.1055/a-1594-1833

In the above-mentioned article the first author's affiliation was changed. This was corrected in the online version on 18.5.2022. 